\title{
Establishment of human pancreatic cancer gemcitabine-resistant cell line with ribonucleotide reductase overexpression
}

\author{
CONGFEI WANG ${ }^{1}$, WEIWEI ZHANG ${ }^{2}$, MINGJUAN FU ${ }^{2}$, AIQIN YANG $^{2}$, HEGUANG HUANG $^{1}$ and JIEMING XIE ${ }^{2}$ \\ ${ }^{1}$ Department of General Surgery, Fujian Medical University Union Hospital, Fuzhou, Fujian 350001; \\ ${ }^{2}$ Department of Pharmacology, Fujian Medical University, Fuzhou, Fujian 350004, P.R. China
}

Received May 31, 2014; Accepted August 29, 2014

DOI: $10.3892 /$ or.2014.3599

\begin{abstract}
A gemcitabine (GEM)-resistant human pancreatic cancer cell line (PANC-1RG7) was established in vitro by gradually increasing GEM concentrations and cloning cell cultures to develop a cellular model of acquired drug resistance studies. We found that PANC-1RG7 cells exhibited significantly different morphological characteristics from parental cells. PANC-1RG7 cells grew slowly $(\mathrm{p}<0.05)$, yet the cell cycle remained unchanged $(\mathrm{p}>0.05)$. PANC-1RG7, with a resistance index to GEM of 39.9, showed cross-resistance characteristics to methotrexate, gefitinib, cisplatin and 5-fluorouracil. The proliferation inhibition of GEM was significantly reduced in vivo $(\mathrm{p}<0.05)$. The known resistance-associated genes and proteins we detected remained unchanged, with the exception of cytidine deaminase, multidrug resistance-related protein and breast cancer resistance protein genes, which decreased; by contrast, 5'-nucleotidase, ribonucleotide reductase (RRM) 1 and RRM2 proteins increased $(\mathrm{p}<0.05)$. Therefore, a cell line with acquired GEM resistance was established successfully. Resistance was acquired by overexpressing RRM1 and RRM2 proteins.
\end{abstract}

\section{Introduction}

Pancreatic cancer is a common digestive malignant tumor with low resection rate, high mortality rate and poor prognosis, as the characteristics of this tumor are masked. Pancreatic cancer patients who cannot undergo surgery are subjected to chemotherapy as a fundamental treatment modality; this modality is also a key component of systemic therapy (1).

Correspondence to: Professor Heguang Huang, Department of General Surgery, Fujian Medical University Union Hospital, 29 Xinquan Road, Fuzhou, Fujian 350001, P.R. China

E-mail: hhuang2@aliyun.com

Professor Jieming Xie, Department of Pharmacology, Fujian Medical University, 88 Jiaotong Road, Fuzhou, Fujian 350004, P.R. China

E-mail: jmxie@mail.fjmu.edu.cn

Key words: pancreatic cancer, PANC-1 cell line, gemcitabine, resistance, ribonucleotide reductase
In pancreatic cancer chemotherapy, gemcitabine (GEM) was initially recommended as a first-line drug by the Food and Drug Administration (USA) in 1997. Since then, research on combination chemotherapies, such as cytotoxic drugs [5-fluorouracil (2), cisplatin (3) and capecitabine (4)] and biological agents [erlotinib (5), cetuximab (6) and bevacizumab (7)], as second-line modes of chemotherapy has been extensively conducted. Although GEM is currently the preferred drug for single chemotherapeutic applications in pancreatic cancer, the inherent and acquired resistance of cancer cells to GEM prevents the efficient improvement of the clinical benefit and survival of patients. Furthermore, the efficiency of this treatment is very low (12\%) (8); as such, this drawback should be resolved in clinical applications. However, related studies have shown that the prognosis of pancreatic cancer in the past 10 years has remained unchanged.

The resistance to GEM is induced by several factors. Although numerous mechanisms have been presented, the main mechanism remains unclear. This resistance is affected by several key molecular factors, including deficiencies in drug uptake, activation of DNA repair pathways, resistance to apoptosis, enhancement of tumor microenvironments, overexpression of signaling proteins, mutations in kinase domains, activation of alternative pathways, mutations of genes and conversion to an epithelial-mesenchymal transition-like phenotype. Hence, GEM-resistance mechanisms involved in pancreatic cancer should be investigated; furthermore, a highly efficient multitarget drug with low toxicity should be developed to synergize current chemotherapy drugs or reverse drug resistance for pancreatic cancer treatment. The present study was conducted to establish a human pancreatic cancer GEM-resistant cell line and determine its biological characteristics for future studies.

\section{Materials and methods}

Cell culture and animal feeding. Human pancreatic cancer cell line PANC-1 was purchased from the Shanghai Institute of Cell Biology, China. These cells were incubated with RPMI- $1640+10 \%$ fetal bovine serum at $37^{\circ} \mathrm{C}$ in a cell incubator with $5 \% \mathrm{CO}_{2}$ and then digested with $0.25 \%$ trypsinogen $+2 \%$ ethylenediaminetetraacetic acid for passage at a ratio of 1:2-4 once at an interval of 2-3 days. Male nude mice were obtained from the Animal Center of the Peking Union Medical College, China. The mice were fed in a specific pathogen free-grade 
animal room at the Fujian Medical University Animal Center in strict accordance with aseptic principles. GEM (Hengda Pharmaceutical Co., Ltd., Shanxi, China) was dissolved in normal saline to obtain a final concentration of $100 \mathrm{mmol} / \mathrm{l}$ and stored at $-20^{\circ} \mathrm{C}$.

Establishment of human pancreatic cancer GEM-resistant cell line. To develop GEM-resistant PANC-1 cell line, we exposed the cells to increasing concentrations of GEM (from $50 \mathrm{nmol} / 1$ to $2 \mu \mathrm{mol} / \mathrm{l}$ ) with repeated subcultures until the cells became fully resistant to GEM. Subsequently, the cells in the logarithmic phase (1/well x $50 \mu \mathrm{l})$ were seeded in 96-well culture plates containing $50 \mu \mathrm{l}$ of supernatant liquid. This liquid had been used to incubate fresh mouse spleen cells for 4 days and then incubated the pancreatic cancer cells for 2 weeks to prepare the cloning culture. Single cell colonies were selected by GEM. After the cultures were cloned thrice, a stable cell clone termed PANC-1RG7 with a uniformly resistant mechanism was obtained.

Morphology and ultrastructure. Cell size and the contours of PANC-1 and PANC-1RG7 cells were observed under an optical microscope. To observe ultramicrostructure characteristics, we harvested $2 \times 10^{6}$ cells and washed them thrice with phosphate-buffered saline (PBS). Subsequently, the cells were fixed in ice-cold $4 \%$ glutaraldehyde for $2 \mathrm{~h}$. The samples were subsequently fixed in $1 \%$ osmic acid for $2 \mathrm{~h}$, gradually dehydrated with acetone and embedded in epoxy resin. The cells were then observed under a transmission electron microscope.

Cell growth curve. PANC-1 and PANC-1RG7 cells in the logarithmic phase $(5,000 /$ well $\mathrm{x} 1 \mathrm{ml})$ were seeded in 24 -well culture plates. After $24 \mathrm{~h}$ of attachment, the cells were harvested and counted under an inverted microscope with $0.2 \%$ trypan blue dye. Three-wells of each cell line were monitored daily for 12 days. The cell growth curves of the 2 cell lines were drawn, and doubling time was calculated using the following equation: $T_{\mathrm{d}}=t \times 24 \mathrm{~h} \times[\lg 2 /(\lg N t-\lg N o)]$, where No is the number of cells when the logarithmic growth phase began, Nt is the number of cells before cell death occurred, and $t$ is the time between the 2 phases. Each experiment was repeated thrice.

Cell cycle analysis by flow cytometry. PANC-1 and PANC-1RG7 cells $\left(1 \times 10^{6}\right)$ were harvested, washed thrice with PBS and fixed in ice-cold $75 \%$ alcohol for $>12 \mathrm{~h}$. After fixation was completed, samples were stained with $0.005 \%$ propidium iodide for $30 \mathrm{~min}$ in the dark at room temperature, and then analyzed to determine the DNA content by FACSCalibur (Becton-Dickinson, Mountain View, CA, USA). Each experiment was repeated thrice.

Sulforhodamine B (SRB) assays. PANC-1 (1,000/well x $100 \mu \mathrm{l})$ and PANC-1RG7 (1,500/well x $100 \mu \mathrm{l})$ cells in the logarithmic phase were seeded in 96-well culture plates and incubated for $24 \mathrm{~h}$ until adherence occurred. Then, cells were treated with different concentrations of GEM, adriamycin (ADM), mitomycin C (MMC), paclitaxel (PTX), methotrexate (MTX), vincristine (VCR), gefitinib (GEF), cisplatin (DDP) and 5-fluorouracil (5-FU). Control cells were supplemented with $100 \mu 1$ of RPMI-1640 culture medium. The treated cells were incubated with drugs for $96 \mathrm{~h}$ before SRB assays described previously (9). The dose-effect curve was plotted to calculate $50 \%$ inhibitory concentration $\left(\mathrm{IC}_{50}\right)$ and resistance index $(\mathrm{RI})$. Each experiment was repeated thrice.

Establishment of animal models and drug intervention. After permission of Fujian Medical University Laboratory Animal Welfare \& Ethics Committee, PANC-1 and PANC-1RG7 cells ( $5 \times 10^{6}$ cells suspended in $200 \mu 1$ of RPMI-1640) were injected percutaneously using a 29-gauge syringe with a hypodermic needle on the right shoulder back of the mice. A total of 40 integrated mice (20 in each cell line) with tumors grown to a final size of $\sim 0.4 \mathrm{~cm}$ in diameter were divided into 4 groups: 10 mice with PANC-1 and 10 mice with PANC-1RG7 were included in the negative control group (injected intraperitoneally with $10 \mathrm{ml} / \mathrm{kg}$ normal saline at 15, 18, 21, 24, 27 and 30 days); 10 mice with PANC-1 and 10 mice with PANC-1RG7 were included in the GEM intervention group (injected intraperitoneally with $50 \mathrm{mg} / \mathrm{kg}$ GEM at 15, 18, 21, 24, 27 and 30 days). We observed the general conditions of the mice and tumors after they were sacrificed at 33 days.

Quantitative real-time polymerase chain reaction analysis $(q P C R)$. PANC-1 and PANC-1RG7 cells $\left(3 \times 10^{6}\right)$ were harvested. Total RNA was extracted and subjected to first-strand complementary DNA as previously described (9). qPCR was performed using the ABI prism 7500 HT sequence detection system (Applied Biosystems, Foster City, CA, USA) to detect the mRNA expression of deoxycytidine kinase (dCK), 5'-nucleotidase (NT5), cytidine deaminase (CDA), equilibrative nucleoside transporter 1 (ENT1), ENT2, ribonucleotide reductase 1 (RRM1), RRM2, DNA polymerase A (POLA), multidrug resistance protein 1 (MDR1), multidrug resistance-related protein (MRP) and breast cancer resistance protein (BCRP). The forward and reverse primers we designed are shown in Table I. Relative expression was calculated using the $\Delta \Delta \mathrm{Ct}$ method and our result passed the validation experiment. The results of control and treated cells are expressed as an average of the triplicate samples of at least 3 independent experiments.

Western blotting. PANC-1 and PANC-1RG7 $\left(9 \times 10^{6}\right)$ cells were harvested. Total protein fractions were extracted, separated on SDS-PAGE and then exposed to specific antibodies using western blotting described earlier (9). The specific primary antibodies we used were mouse monoclonal antibodies anti-human $\beta$-actin (sc-47778), ENT1 (sc-377283), ENT2 (sc-373871), NT5 (sc-32299), POLA (sc-137021), p-gp (sc-55510) (all from Santa Cruz, USA), and MRP (no. ab32574; Abcam, USA); rabbit polyclonal antibodies anti-human DCK (no. ab151966; Abcam, USA), CDA (sc-134754), BCRP (sc-130933) (both from Santa Cruz), Akt (no. BS1810) and mTOR (no. BS3611) (both from BioWorld, USA); rabbit monoclonal antibodies anti-human PI3K (no. 4249; Cell Signaling Technology, USA); and goat polyclonal antibodies anti-human RRM1 (sc-11733) and RRM2 (sc-10846) (both from Santa Cruz). Images were analyzed using Quantity One 4.62. Each experiment was repeated $>3$ times.

Statistical analysis. Experimental data are presented as the means \pm standard deviation (SD) and analyzed by SPSS 19.0. 
Table I. Sequences of polymerase chain reaction primers and sequence-specific probes of target genes and $\beta$-actin.

\begin{tabular}{lllr}
\hline Gene & \multicolumn{1}{c}{ Forward primer (5'-3') } & \multicolumn{1}{c}{ Reverse primer (5'-3') } & $\begin{array}{c}\text { Product length } \\
\text { (bp) }\end{array}$ \\
\hline dCK & ATCCAGCTTCCTTCTGTCATTCC & CAACGAAGTGAGAGGCACCAG & 80 \\
NT5 & TAATGGTATAAACACAGGATACCATCCT & CATTATCTACTACAGCTTGCTACCTGACT & 85 \\
CDA & GAAGCGTCCTGCCTGCA & CTGGACCGTCATGACAATATACG & 382 \\
ENT1 & TCTTCATGGCTGCCTTTGC & GGCTTCACTTTCTTGGGCC & 79 \\
ENT2 & CAAGACCTCATGGAAAGGGTG & CCACTCTGAACCCTCTGGTCA & 124 \\
RRM1 & GGCACCCCGTATATGCTCTA & CCAGGGAAGCCAAATACAA & 148 \\
RRM2 & GGCTCAAGAAACGAGGACTG & TCAGGCAAGCAAAATCACAG & 93 \\
POLA & GGCTCGGATCTGTGAACCAA & GGGCTCCATATCTGTTCCCG & 256 \\
MDR1 & AGGTTCCAGGATTGGCGTCTT & CCAGTCATTGCTGCGGTTTCA & 156 \\
MRP & GCGAGTGTCTCCCTCAAACG & TCCTCACGGTGATGCTGTTC & 118 \\
BCRP & GATATGGATTTACGGCTTTGC & CGATGCCCTGCTTTACCAA & 135 \\
$\beta$-actin & AGTGTGACGTGGACATCCGCAAAG & ATCCACATCTGCTGGAAGGTGGAC & 220 \\
\hline
\end{tabular}

dCK, deoxycytidine kinase; NT5, 5'-nucleotidase; CDA, cytidine deaminase; ENT1, equilibrative nucleoside transporter 1; RRM1, ribonucleotide reductase 1; POLA, DNA polymerase A; MDR1, multidrug resistance protein 1; MRP, multidrug resistance-related protein; BCRP, breast cancer resistance protein.

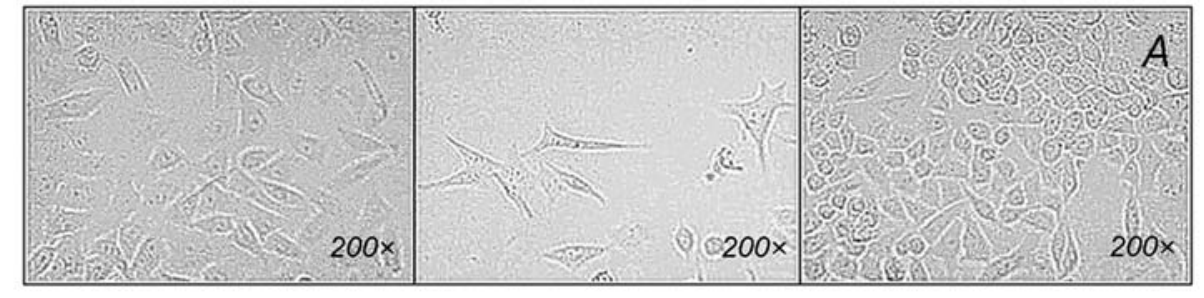



PANC-1

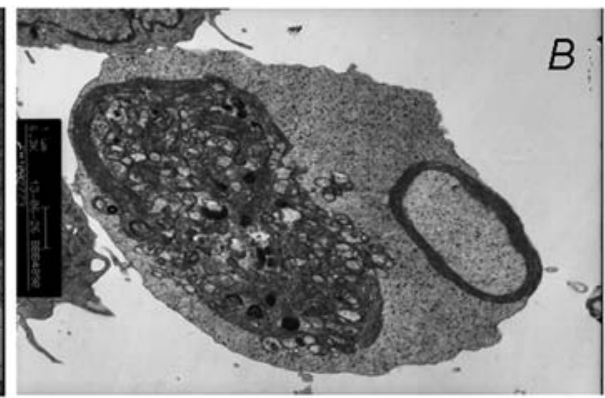

PANC-1RG7

Figure 1. (A) Morphological changes in pancreatic cancer PANC-1 cells before, during and after GEM intervention under an optical microscope. (B) Ultrastructural changes in pancreatic cancer PANC-1 and PANC-1RG7 cells under a transmission electron microscope. GEM, gemcitabine.

Comparisons were performed using Student's t-test between 2 groups. $\mathrm{P}<0.05$ was considered to indicate a statistically significant difference.

\section{Results}

Morphological and ultrastructure characteristics. PANC-1 cells appeared fusiform under an optical microscope at a magnification of x200. During GEM intervention, the cells appeared polygonal with elongated pseudopodia and growth retardation; the size significantly increased and numerous vacuoles were formed in the cytoplasm. Cell growth was gradually restored after GEM was removed; a fusiform was formed but remained smaller and grew more slowly than parental cells (Fig. 1A).

PANC-1 cells contained intact a cell membrane and nucleus, numerous microvilli on the membrane, abundant organelles and a satisfactory state under a transmission electron microscope. However, PANC-1RG7 exhibited different ultrastructural characteristics. In particular, small vacuoles and lipid droplets 


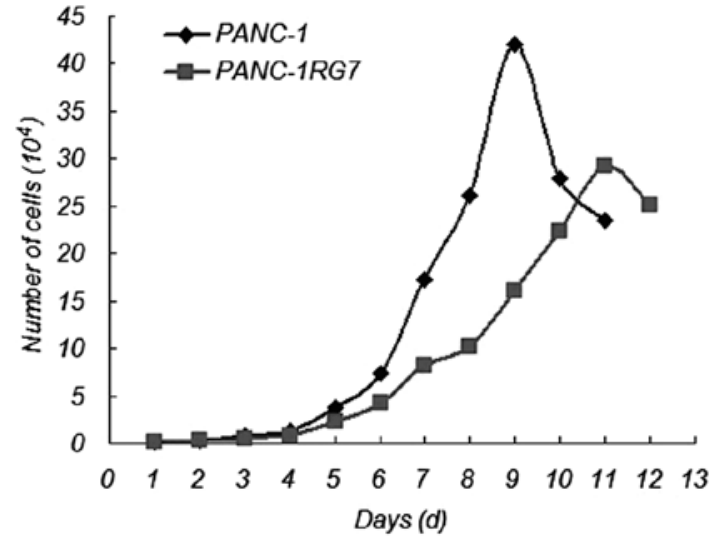

Figure 2. Cell growth curves of PANC-1 and PANC-1RG7 cells. PANC-1RG7 cells grew significantly more slowly than the parental PANC-1 cells.

were formed in the cytoplasm. The number of glycogen granules and lysosomes increased significantly, the mitochondrial cristae was broken as vacuolization occurred, and the rough endoplasmic reticulum became swollen (Fig. 1B).

Cell growth curve. Compared with the parental cell PANC-1, GEM-resistant pancreatic cancer PANC-1RG7 cells slowly grew at a significant rate (Fig. 2). The doubling times of PANC-1 and PANC-1RG7 cells were $25.83 \pm 2.03$ and $33.83 \pm 2.15 \mathrm{~h}$, respectively. The doubling time of PANC-1RG7 cells was significantly increased $(\mathrm{p}<0.05)$.

Cell cycle analysis by flow cytometry. In PANC-1, 68.98 \pm 2.32 and $18.02 \pm 0.63 \%$ of the cells were detected in the $\mathrm{G} 0 / \mathrm{G} 1$ and S phase, respectively. In PANC-1RG7, 69.23 \pm 3.03 and $17.77 \pm 0.89 \%$ of the cells were detected in the G0/G1 and $\mathrm{S}$ phase, respectively. No significant difference was determined (p $>0.05$; Fig. 3 ).

SRB assays. We detected 9 common chemotherapeutics, including GEM, ADM, MMC, PTX, MTX, VCR, GEF, DDP and 5-FU. The $\mathrm{IC}_{50}$ values of GEM, MTX, GEF, DDP and 5-FU were statistically different between PANC-1 and PANC-1RG7; by contrast, the $\mathrm{IC}_{50}$ values of the other drugs were not different (Table II). The RIs of GEM, MTX, GEF, DDP and 5-FU were 39.9, 2.24, 1.42, 2.35 and 7.00, respectively. This result indicated that the PANC-1RG7 cells established in this study expressed resistance to GEM and cross-resistance to MTX, GEF, DDP and 5-FU.

Establishment of animal models and drug intervention. We successfully established nude mouse subcutaneous tumor models. The mice were sacrificed at 33 days. Subcutaneous tumors were completely peeled off and weighed (Table III). PANC-1 tumors in the negative control group were significantly smaller than PANC-1RG7 tumors $(\mathrm{p}<0.05)$. This result indicated that PANC-1RG7 cells grew faster in vivo than PANC-1 cells. A significant difference was observed in tumor weights before and after GEM intervention was administered in PANC-1 $(p<0.05)$, but not in PANC-1RG7 $(p<0.05)$. The inhibition rates of GEM in PANC-1 and PANC-1GR7 were



PANC-1

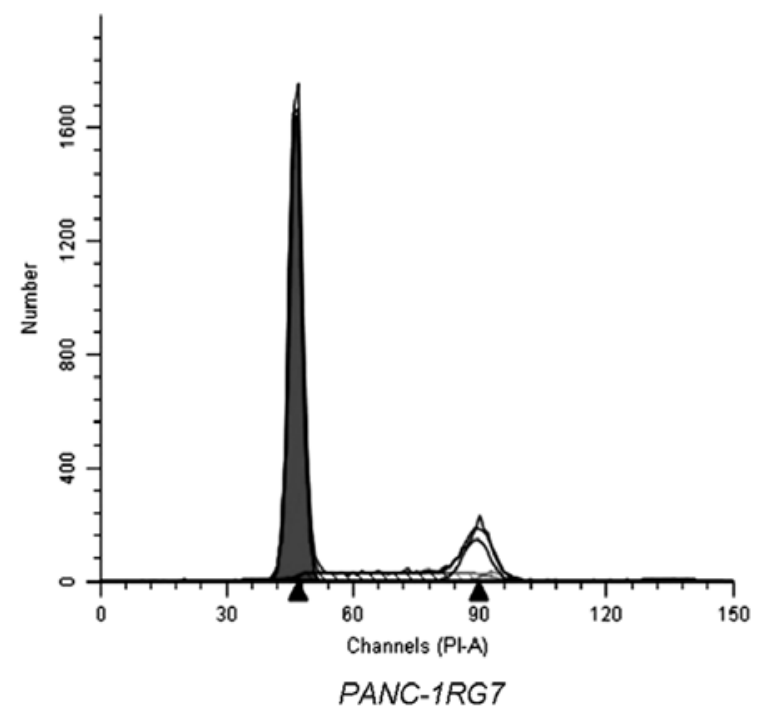

Figure 3. Flow cytometric analysis of the cell cycle. No significant difference was observed between PANC-1 and PANC-1RG7.

82.03 and $33.40 \%$, respectively. This finding indicated that the inhibition of GEM decreases in vivo.

qPCR. We examined the expression levels of dCK, NT5, CDA, ENT1, ENT2, RRM1, RRM2, POLA, MDR1, MRP and BCRP at mRNA levels by qPCR. However, only CDA, MRP and BCRP expressions changed at mRNA levels. The expression levels of these 3 genes in PANC-1RG7 were lower than those in PANC-1 with a significant difference (Table IV).

Western blotting. We further examined the expression levels of dCK, NT5, CDA, ENT1,ENT2, RRM1, RRM2, POLA, MDR1, MRP and BCRP at protein levels by western blotting. The key components of the PI3K/Akt/mTOR signaling pathway were also determined. The examined proteins, except BCRP, were expressed in PANC-1 and PANC-1RG7. Compared with those in parental PANC-1, NT5, RRM1 and RRM2 expression levels were significantly increased in PANC-1RG7 $(\mathrm{p}<0.05)$. No changes in other proteins at a protein level were noted (Fig. 4). 
Table II. SRB assay results of PANC-1 and PANC-1RG7 human pancreatic cancer cells treated with various concentrations of GEM, ADM, MMC, PTX, MTX, VCR, GEF, DDP and 5-FU for $96 \mathrm{~h}$.

\begin{tabular}{lccc}
\hline $\mathrm{IC}_{50}$ & \multicolumn{1}{c}{ PANC-1 } & PANC-1RG7 & P-value \\
\hline GEM $(\mu \mathrm{mol} / \mathrm{l})$ & $0.0081 \pm 0.0014$ & $0.3233 \pm 0.0933$ & $0.0023^{\mathrm{a}}$ \\
$\mathrm{ADM}(\mathrm{nmol} / \mathrm{l})$ & $10.2380 \pm 2.4875$ & $9.1433 \pm 2.4533$ & 0.7011 \\
MMC $(\mu \mathrm{mol} / \mathrm{l})$ & $0.5689 \pm 0.5180$ & $0.2545 \pm 0.1543$ & 0.4972 \\
PTX $(\mathrm{nmol} / \mathrm{l})$ & $2.2618 \pm 0.2262$ & $2.4840 \pm 0.1500$ & 0.2682 \\
MTX $(\mathrm{nmol} / \mathrm{l})$ & $0.9346 \pm 0.1649$ & $2.0948 \pm 0.1672$ & $0.0199^{\mathrm{b}}$ \\
VCR $(\mathrm{nmol} / \mathrm{l})$ & $7.0513 \pm 2.3578$ & $5.4185 \pm 1.7090$ & 0.4265 \\
GEF $(\mu \mathrm{mol} / \mathrm{l})$ & $7.2575 \pm 0.5216$ & $10.281 \pm 0.2890$ & $0.0189^{\mathrm{b}}$ \\
DDP $(\mu \mathrm{mol} / \mathrm{l})$ & $0.6317 \pm 0.2159$ & $1.4853 \pm 0.4649$ & $0.0108^{\mathrm{b}}$ \\
5-FU $(\mu \mathrm{mol} / \mathrm{l})$ & $1.9298 \pm 0.4420$ & $13.509 \pm 2.7563$ & $0.0001^{\mathrm{a}}$
\end{tabular}

Data shown are $\mathrm{IC}_{50}($ mean $\pm \mathrm{SD}) .{ }^{\mathrm{a} C}$ Compared with PANC- $1, \mathrm{p}<0.01 ;{ }^{\mathrm{b}}$ compared with PANC $-1, \mathrm{p}<0.05$. GEM, gemcitabine; ADM, adriamycin; MMC, mitomycin C; PTX, paclitaxel; MTX, methotrexate; VCR, vincristine; GEF, gefitinib; DDP, cisplatin; 5-FU; 5-fluorouracil; SRB, sulforhodamine B.

Table III. Subcutaneous tumor weight of each group of mice with pancreatic cancer $(\mathrm{n}=10$, mean $\pm \mathrm{SD})$.

\begin{tabular}{lcc}
\hline Tumor weight $(\mathrm{g})$ & Control & GEM $(50 \mathrm{mg} / \mathrm{kg})$ \\
\hline PANC-1 & $0.2118 \pm 0.0521$ & $0.0381 \pm 0.0215^{\mathrm{a}}$ \\
PANC-1RG7 & $0.3247 \pm 0.1292^{\mathrm{b}}$ & $0.2163 \pm 0.0833$
\end{tabular}

Subcutaneous tumors were completely peeled off after the mice were sacrificed at 33 days. ${ }^{a}$ Compared with control group, $\mathrm{p}<0.05$; ${ }^{\mathrm{b}} \mathrm{com}-$ pared with PANC-1, p $<0.05$. GEM, gemcitabine.

\section{Discussion}

Chemotherapy is the fundamental treatment modality for pancreatic cancer patients who are unable to undergo surgery; this modality is also a key component of systemic therapy (1). GEM is currently the preferred drug for the treatment of pancreatic cancer by single chemotherapeutic applications. However, the inherent and acquired resistance of cancer cells to GEM limits its efficiency. Thus far, no effective drug has improved the clinical benefits of GEM. Hence, resistance to GEM remains a vital problem. Numerous mechanisms have been presented, however, the main one remains unclear. In the present study, a stable human pancreatic cancer GEM-resistant cell line was established for use in further studies on GEM resistance.

Intermittent intervention in gradually increasing concentration or pulse intervention in large concentrations can be performed to establish drug-resistant cancer cell lines. The former method can be used to simulate the intermittent administration of drugs in clinical applications with a high achievement ratio and increased stability. Although this method requires time-consuming procedures, we performed this method in the present study. In China, human pancreatic cancer cell line SW1990, which is derived from pancreatic cancer accompanied by metastatic spleen, is commonly
Table IV. mRNA expression of dCK, NT5, CDA, ENT1, ENT2, RRM1, RRM2, POLA, MDR1, MRP and BCRP by qPCR.

\begin{tabular}{lcc}
$\begin{array}{l}\text { PANC-1RG7 } \\
\text { gene }\end{array}$ & $\Delta \Delta \mathrm{Ct}$ & $2^{-\Delta \Delta \mathrm{Ct}}$ \\
\hline dCK & $0.06 \pm 0.52$ & $1.00 \pm 0.33$ \\
NT5 & $0.62 \pm 1.33$ & $0.86 \pm 0.77$ \\
CDA & $5.88 \pm 0.69$ & $0.02 \pm 0.01^{\mathrm{a}}$ \\
ENT1 & $0.70 \pm 0.22$ & $0.62 \pm 0.09$ \\
ENT2 & $0.38 \pm 0.68$ & $0.83 \pm 0.36$ \\
RRM1 & $0.04 \pm 0.43$ & $1.00 \pm 0.29$ \\
RRM2 & $-0.04 \pm 0.40$ & $1.05 \pm 0.30$ \\
POLA & $0.45 \pm 1.55$ & $0.98 \pm 0.67$ \\
MDR1 & $1.27 \pm 1.44$ & $0.55 \pm 0.46$ \\
MRP & $1.48 \pm 0.28$ & $0.36 \pm 0.07^{\mathrm{a}}$ \\
BCRP & $2.42 \pm 0.34$ & $0.19 \pm 0.05^{\mathrm{a}}$
\end{tabular}

PANC-1 and PANC-1RG7 human pancreatic cancer cells were harvested and total RNA was extracted. Only the mRNA expression of CDA, MRP and BCRP decreased in PANC-1RG7 $\left(2^{-\Delta \Delta C t}<0.50\right)$. ${ }^{\text {a }}{ }^{-\Delta \Delta \mathrm{Ct}}<0.5$. dCK, deoxycytidine kinase; NT5, 5'-nucleotidase; CDA, cytidine deaminase; ENT1, equilibrative nucleoside transporter 1; RRM1, ribonucleotide reductase 1; POLA, DNA polymerase A; MDR1, multidrug resistance protein 1; MRP, multidrug resistance-related protein; BCRP, breast cancer resistance protein.

used to establish a pancreatic cancer GEM-resistant cell line (10-12), while MIA PaCa-2 derived from pancreas tissues is used in other countries (13-15). However, we chose human pancreatic cancer cell line PANC-1, which is derived from pancreatic ductal carcinoma. Commonly used in clinical practice, this cell line is highly sensitive to GEM owing to its low differentiation. The stable cell clone termed PANC-1RG7 with a uniform resistant mechanism was obtained after GEM intervention was conducted for 2 years and clone cultures were 

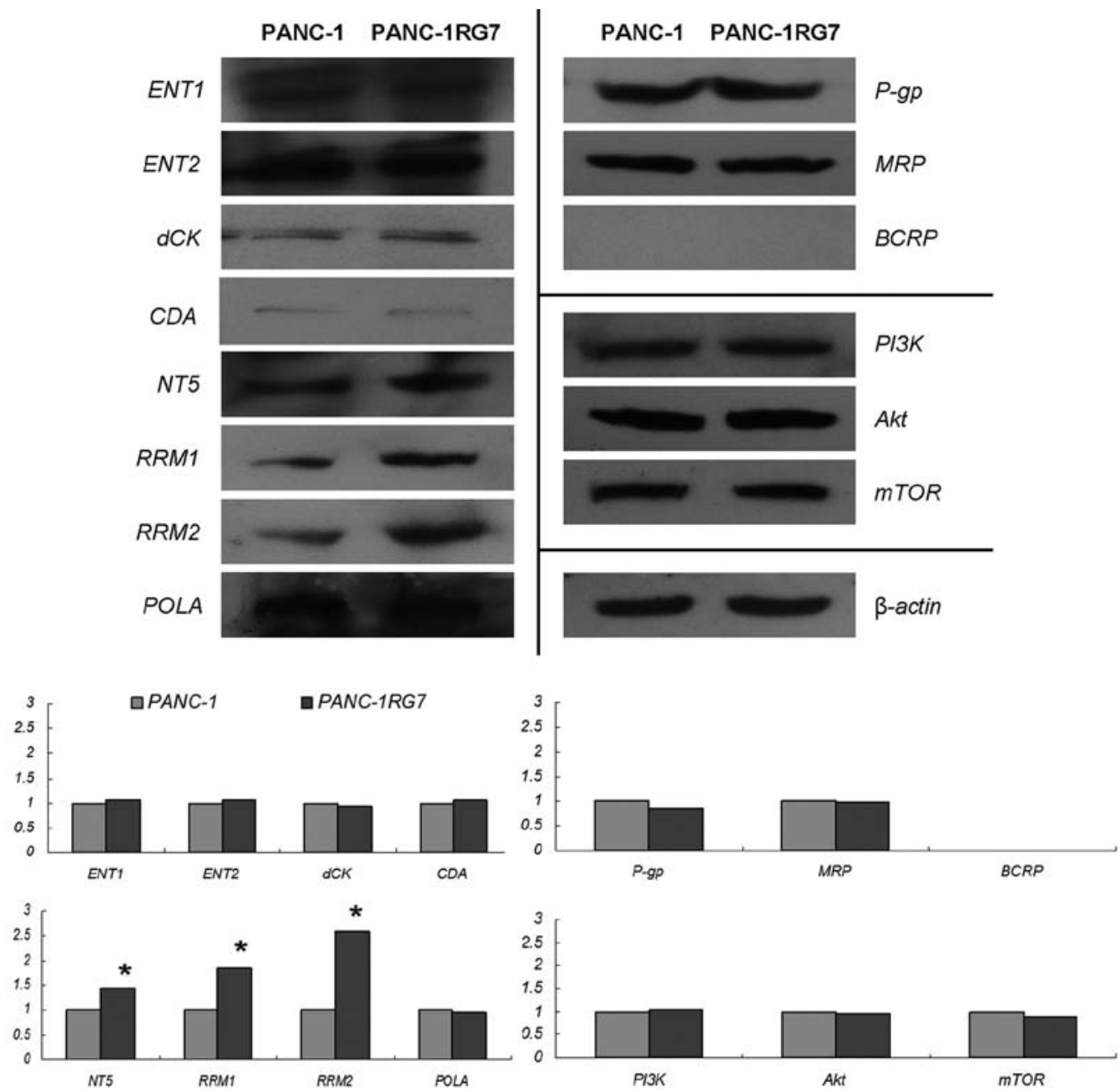

Figure 4. Western blotting for the resistance-related factors. The examined proteins, except BCRP, were expressed in PANC-1 and PANC-1RG7. Compared with those of the parental PANC-1 cells, NT5, RRM1 and RRM2 expression levels were significantly increased in PANC-1RG7 (p<0.05). No changes in other proteins at the protein level were noted. Results represent 3 independent experiments. Gray scales shown at the bottom indicate results compared with $\beta$-actin.

prepared thrice. Our results showed that the RI of GEM was 39.9, indicating low resistance.

Changes in the morphological characteristics of resistant cells indicate acquired resistance. The established GEM-resistant cells were smaller and grew more slowly than the parental cells. Cell organs, such as lysosomes, mitochondria and rough endoplasmic reticulum, significantly changed, as observed under a transmission electron microscope. These changes may be considered the basis of functional changes related to resistance mechanisms. Multidrug resistance (16), characterized by cells that are resistant not only to intervention drug but also to other chemotherapeutics without structural or functional relationships existed in PANC-1RG7 with crossresistance to MTX, GEF, DDP and 5-FU, suggesting that a common mechanism could be implicated in this resistance. As such, in vivo studies involving xenografts are necessary to detect biological behavior and tumor characterization. The established PANC-1RG7 cells indicated an increased discernible invasion and growth compared with parental PANC-1 cells; by contrast, in vitro studies showed a slow growth. Further studies should be conducted to investigate the possible mechanism implicated in the difference between in vivo and in vitro processes.
ENTs facilitate the entry of GEM, a pyrimidine analog, across the plasma membrane of cells. GEM is then phosphorylated intracellularly by $\mathrm{dCK}$ via multiple steps to derive diphosphate and triphosphate. The former is an active metabolite inhibiting RRM, resulting in a decrease in intracellular dCTP; thus, DNA synthesis is suppressed. The latter inhibits DNA synthesis by interfering with the incorporation of endogenous dCTP into DNA. Studies on GEM resistance mechanisms have shown that factors involved in GEM metabolism and transport are related to resistance. In the present study, the expression levels of the main factors in PANC-1RG7 and parental PANC-1 were detected. CDA expression at an mRNA level significantly decreased in PANC-1RG7, yet it remained unchanged at a protein level. The expressions of NT5, RRM1 and RRM2 proteins were significantly increased in PANC-1RG7 compared with those in PANC-1RG7. By contrast, no change was observed at the mRNA level. No linear relationship between mRNA and protein expression was noted since mRNA undergoes a series of regulatory processes, including microRNA regulation, translation, post-translational modification (e.g., glycosylation and phosphorylation), and protein transport, to express proteins. Thus, the differences in the changes between mRNA and protein expression could 
be acceptable. As active proteins, enzymes are involved in activities more directly related to expressions at protein levels. Hence, the overexpression of NT5, RRM1 and RRM2 was necessary to induce the resistance of the established PANC-1RG7 to GEM.

Increased activities of RRM1 and RRM2 possibly promote the conversion of nucleoside to deoxynucleoside and accelerate DNA polymerization and repair, resulting in resistance (17). In GEM metabolism and transport, 4 factors, ENT1, dCK, RRM1 and RRM2, are implicated in acquired resistance (18). As ENT1 expression decreases, GEM intake is reduced and cytotoxicity is decreased in vivo (19). The deficiency in dCK activities is one of the mechanisms by which pancreatic cancer cells develop resistance to chemotherapeutic drugs (20) since $\mathrm{dCK}$ is an important factor in the intracellular conversion of GEM to an active metabolite. However, ENT1 and dCK expression in PANC-1RG7 remained unchanged at the mRNA and protein levels. Enzyme activity is not only affected by mRNA or protein expression; studies have shown that dCK activity, protein and gene expression levels are significantly correlated (21). No dCK activity was detected directly due to limited experimental conditions. However, dCK failed to induce PANC-1RG7 to develop resistance to GEM. In a previous study, the overexpression of NT5 was observed in a human pancreatic cancer GEM-resistant cell line (14); however, further studies should be conducted to determine whether or not this overexpression increases the removal of GEM.

P-gp, MRP and BCRP are 3 multidrug-resistant proteins relevant to tumor stem cell. On the basis of the results of expression detection, we found that P-gp and MRP proteins were expressed in human pancreatic cancer PANC-1 cells; MDR1, MRP and BCRP genes were also expressed in PANC-1 cells, indicating the inherent resistance of PANC-1. The expressions did not increase after GEM intervention was administered in the present study, and the gene expression of MRP and BCRP decreased. However, studies have yet to determine whether or not the resistance of cancer cells to chemotherapeutics is attributed to the decrease in the gene expression of MRP and BCRP. Further studies are required to determine if these changes are correlated with cell resistance. Nevertheless, the proteins and genes not implicated in the resistance of PANC-1RG7 to GEM could be identified. Moreover, changes in apoptotic signaling pathway are related to the resistance of pancreatic cancer cells. Thus, apoptosis-regulatory proteins are abnormally expressed in pancreatic cancer cells (22). No changes in PI3K, Akt and mTOR protein expression were observed in PANC-1RG7.

In summary, human pancreatic cancer GEM-resistant cell line PANC-1RG7 was established in this study. These cells grew slowly in vitro but rapidly in vivo. In vitro and in vivo experimental results showed that PANC-1RG7 exhibited stable resistance to GEM and cross-resistance to other chemotherapeutics, such as MTX, GEF, DDP and 5-FU. Of all the factors related to GEM resistance, only RRM1 and RRM2 protein expression increased in the resistant cells, thereby inducing resistance to GEM. This result indicated that the overexpression of RRM1 and RRM2 was necessary to induce the resistance of PANC-1RG7 to GEM.

Cancer-resistant cell lines established in vitro remain the main tools used to study the mechanisms of acquired tumor resistance. The established pancreatic cancer GEM-resistant cell line PANC-1RG7 may also be used as an important tool to investigate the acquired resistance of pancreatic cancer, RRM, or new chemotherapy drugs that can reverse GEM resistance.

\section{Acknowledgements}

The present study was supported by the National Science Foundation of China (no. 30772587), the Great Research Project of Fujian Medical University (no. 09ZD012), and the Natural Science Foundation of Fujian Province (nos. C0510012 and 2011J01188).

\section{References}

1. El Maalouf G, Le Tourneau C, Batty GN, Faivre S and Raymond E: Markers involved in resistance to cytotoxics and targeted therapeutics in pancreatic cancer. Cancer Treat Rev 35: $167-174,2009$.

2. Berlin JD, Catalano P, Thomas JP, Kugler JW, Haller DG and Benson AB III. Phase III study of gemcitabine in combination with fluorouracil versus gemcitabine alone in patients with advanced pancreatic carcinoma: Eastern Cooperative Oncology Group Trial E2297. J Clin Oncol 20: 3270-3275, 2002.

3. Heinemann V, Quietzsch D, Gieseler F, Gonnermann M, Schönekäs H, Rost A, Neuhaus H, Haag C, Clemens M, Heinrich B, Vehling-Kaiser U, Fuchs M, Fleckenstein D, Gesierich W, Uthgenannt D, Einsele H, Holstege A, Hinke A, Schalhorn A and Wilkowski R: Randomized phase III trial of gemcitabine plus cisplatin compared with gemcitabine alone in advanced pancreatic cancer. J Clin Oncol 24: 3946-3952, 2006.

4. Bernhard J, Dietrich D, Scheithauer W, Gerber D, Bodoky G, Ruhstaller T, Glimelius B, Bajetta E, Schüller J, Saletti P, Bauer J, Figer A, Pestalozzi BC, Köhne CH, Mingrone W, Stemmer SM, Tàmas K, Kornek GV, Koeberle D, Herrmann R; Central European Cooperative Oncology Group: Clinical benefit and quality of life in patients with advanced pancreatic cancer receiving gemcitabine plus capecitabine versus gemcitabine alone: a randomized multicenter phase III clinical trial - SAKK 44/00-CECOG/PAN.1.3.001. J Clin Oncol 26: 3695-3701, 2008.

5. Moore MJ, Goldstein D, Hamm J, Figer A, Hecht JR, Gallinger S, Au HJ, Murawa P, Walde D, Wolff RA, Campos D, Lim R, Ding K, Clark G, Voskoglou-Nomikos T, Ptasynski M, Parulekar W; National Cancer Institute of Canada Clinical Trials Group: Erlotinib plus gemcitabine compared with gemcitabine alone in patients with advanced pancreatic cancer: a phase III trial of the National Cancer Institute of Canada Clinical Trials Group. J Clin Oncol 25: 1960-1966, 2007.

6. Philip PA, Benedetti J, Corless CL, Wong R, O'Reilly EM, Flynn PJ, Rowland KM, Atkins JN, Mirtsching BC, Rivkin SE, Khorana AA, Goldman B, Fenoglio-Preiser CM, Abbruzzese JL and Blanke CD: Phase III study comparing gemcitabine plus cetuximab versus gemcitabine in patients with advanced pancreatic adenocarcinoma: Southwest Oncology Group-directed intergroup trial S0205. J Clin Oncol 28: 3605-3610, 2010.

7. Kindler HL, Friberg G, Singh DA, Locker G, Nattam S, Kozloff M, Taber DA, Karrison T, Dachman A, Stadler WM and Vokes EE: Phase II trial of bevacizumab plus gemcitabine in patients with advanced pancreatic cancer. J Clin Oncol 23: 8033-8040, 2005.

8. Storniolo AM, Enas NH, Brown CA, Voi M, Rothenberg ML and Schilsky R: An investigational new drug treatment program for patients with gemcitabine: results for over 3000 patients with pancreatic carcinoma. Cancer 85: 1261-1268, 1999.

9. Wang C, Yang A, Zhang B, Yin Q, Huang H, Chen M and Xie J: PANC-1 pancreatic cancer cell growth inhibited by cucurmosin alone and in combination with an epidermal growth factor receptor-targeted drug. Pancreas 43: 291-297, 2014.

10. Niu BZ, Chen G, Li LJ, Wu YD and Zhao YP: Drug resistance and activity changes of thioredoxin reductase in pancreatic cancer cell strain SW1990 induced by gemcitabine. Zhongguo Yi Xue Ke Xue Yuan Xue Bao 27: 606-610, 2005 (In Chinese).

11. Yao J, Feng FY, Lin C, Zhang XY, Fu M, Liang X and Yang Y: The mechanism of resistance to 2',2'-difluorodeoxycytidine (gemcitabine) in a pancreatic cancer cell line. Zhonghua Zhong Liu Za Zhi 27: 721-726, 2005 (In Chinese). 
12. An Y, Yao J, Wei JS, Lu ZP, Cai HH, Dai CC, Qian ZY, Xu ZK and Miao Y: Establish a gemcitabine-resistant pancreatic cancer cell line SW1990/GZ and research the relationship between SW1990/GZ and pancreatic cancer stem cell. Zhonghua Wai Ke Za Zhi 48: 999-1003, 2010 (In Chinese).

13. Togawa A, Ito H, Kimura F, Shimizu H, Ohtsuka M, Shimamura F, Yoshidome H, Katoh A and Miyazaki M: Establishment of gemcitabine-resistant human pancreatic cancer cells and effect of brefeldin-a on the resistant cell line. Pancreas 27: 220-224, 2003.

14. Kazuno H, Sakamoto K, Fujioka A, Fukushima M, Matsuda A and Sasaki T: Possible antitumor activity of 1-(3-C-ethynyl- $\beta$ D-ribo-pentofuranosyl)cytosine (ECyd, TAS-106) against an established gemcitabine (dFdCyd)-resistant human pancreatic cancer cell line. Cancer Sci 96: 295-302, 2005.

15. Kagawa S, Takano S, Yoshitomi H, Kimura F, Satoh M, Shimizu H, Yoshidome H, Ohtsuka M, Kato A, Furukawa K, Matsushita K, Nomura $\mathrm{F}$ and Miyazaki M: Akt/mTOR signaling pathway is crucial for gemcitabine resistance induced by Annexin II in pancreatic cancer cells. J Surg Res 178: 758-767, 2012.

16. Uchiyama-Kokubu $\mathrm{N}$ and Watanabe $\mathrm{T}$ : Establishment and characterization of adriamycin-resistant human colorectal adenocarcinoma HCT-15 cell lines with multidrug resistance. Anticancer Drugs 12: 769-779, 2001.

17. Goan YG, Zhou B, Hu E, Mi S and Yen Y: Overexpression of ribonucleotide reductase as a mechanism of resistance to 2,2-difluorodeoxycytidine in the human KB cancer cell line. Cancer Res 59: 4204-4207, 1999.
18. Nakano Y, Tanno S, Koizumi K, Nishikawa T, Nakamura K, Minoguchi M, Izawa T, Mizukami Y, Okumura T and Kohgo Y: Gemcitabine chemoresistance and molecular markers associated with gemcitabine transport and metabolism in human pancreatic cancer cells. Br J Cancer 96: 457-463, 2007.

19. García-Manteiga J, Molina-Arcas M, Casado FJ, Mazo A and Pastor-Anglada M: Nucleoside transporter profiles in human pancreatic cancer cells: role of hCNT1 in 2',2'-difluorodeoxycytidine-induced cytotoxicity. Clin Cancer Res 9: 5000-5008, 2003.

20. Bergman AM, Pinedo HM and Peters GJ: Determinants of resistance to 2',2'-difluorodeoxycytidine (gemcitabine). Drug Resist Updat 5: 19-33, 2002.

21. Kroep JR, Loves WJ, van der Wilt CL, Alvarez E, Talianidis I, Boven E, Braakhuis BJ, van Groeningen CJ, Pinedo HM and Peters GJ: Pretreatment deoxycytidine kinase levels predict in vivo gemcitabine sensitivity. Mol Cancer Ther 1: 371-376, 2002.

22. Graber HU, Friess H, Zimmermann A, Korc M, Adler G, Schmid R and Büchler MW: Bak expression and cell death occur in peritumorous tissue but not in pancreatic cancer cells. J Gastrointest Surg 3: 74-80, 1999. 